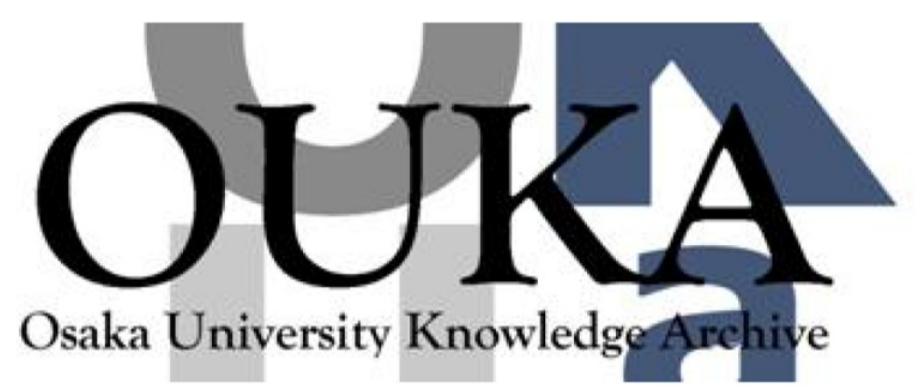

\begin{tabular}{|c|l|}
\hline Title & $\begin{array}{l}\text { Volatility Derivatives and Model-free Implied } \\
\text { Leverage }\end{array}$ \\
\hline Author(s) & Fukasawa, Masaaki \\
\hline Citation & $\begin{array}{l}\text { International Journal of Theoretical and } \\
\text { Applied Finance. 17(1) p. 1450002 }\end{array}$ \\
\hline Issue Date & $2014-02$ \\
\hline oaire:version & AM \\
\hline URL & https://hdl. handle. net/11094/77642 \\
\hline rights & @ copyright World Scientific Publishing Company \\
\hline Note & \\
\hline
\end{tabular}

Osaka University Knowledge Archive : OUKA

https://ir. Library. osaka-u. ac. jp/

Osaka University 


\title{
Volatility Derivatives and Model-free Implied Leverage
}

\author{
Masaaki Fukasawa \\ Department of Mathematics, Osaka University \\ 1-1 Machikaneyama, Toyonaka, Osaka, JAPAN \\ Center for the Study of Finance and Insurance, Osaka University \\ Japan Science and Technology Agency, CREST
}

October 24, 2013

\begin{abstract}
We revisit robust replication theory of volatility derivatives and introduce a broader class which may be considered as the second generation of volatility derivatives. One of them is a swap contract on the quadratic covariation between an asset price and the model-free implied variance (MFIV) of the asset. It can be replicated in a model-free manner and its fair strike may be interpreted as a model-free measure for the covariance of the asset price and the realized variance. The fair strike is given in a remarkably simple form, which enable to compute it from the Black-Scholes implied volatility surface. We call it the model-free implied leverage (MFIL) and give several characterizations. In particular we show its simple relation to the Black-Scholes implied volatility skew by an asymptotic method. Further to get an intuition, we demonstrate some explicit calculations under the Heston model. We report some empirical evidence from the time series of the MFIV and MFIL of the Nikkei stock average.

Keywords: Variance swap; Gamma swap; Leverage effect; Volatility skew; Robust hedging; Asymptotic expansion.
\end{abstract}




\section{Introduction}

Ever since the Chicago Board Options Exchange (CBOE) revised in 2003 their methodology for VIX, a volatility index of the SPX option market, both the new method and an underlying model-free formula for the fair strike of a variance swap have received much attention in the financial industry and have been applied to many other markets in order to meet the demand of financial practitioners for a model-free estimate of the future volatility of the underlying asset price. Let $T>0$ and denote by $S^{1}, S^{0}$, respectively, the market price processes of a risky asset and the risk-free zero coupon bond with maturity $T, S_{T}^{0}=1$. By assuming the discounted process $S=S^{1} / S^{0}$ to be a continuous semi-martingale, a simple application of the Itô-Tanaka formula gives that

$$
\int_{0}^{T} g\left(S_{u}\right) \mathrm{d}\langle\log (S)\rangle_{u}=f_{g}\left(S_{T}\right)-f_{g}\left(S_{0}\right)-\int_{0}^{T} f_{g}^{\prime}\left(S_{u}\right) \mathrm{d} S_{u}
$$

for any locally integrable function $g$, where

$$
f_{g}(s)=2 \int_{1}^{s} \int_{1}^{u} \frac{g(v)}{v^{2}} \mathrm{~d} v \mathrm{~d} u .
$$

Since the last term written as a stochastic integral with respect to $S$ can be replicated by dynamic rebalancing in a self-financing and model-free manner, the option contract with maturity $T$ and payoff given by the left hand side integral is equivalent to the European style option with the same maturity and payoff $f\left(S_{T}\right)-f\left(S_{0}\right)$. The case $g=1$ corresponds to the floating leg of the variance swap, which has zero value at inception (time 0 ) and pays at time $T$ the difference between the floating leg $\langle\log (S)\rangle_{T}$ and a fixed leg (strike) determined at time 0 . The above identity tells in particular that the strike multiplied by $S_{0}^{0}$ must coincide with the price at time 0 of the European option with maturity $T$ and payoff $-2 \log \left(S_{T} / S_{0}\right)$ in order to avoid an arbitrage opportunity. The strike may be interpreted as an expected, or predicted value of $\langle\log (S)\rangle_{T}$. In fact it is the expectation of $\langle\log (S)\rangle_{T}$ under the pricing measure. The idea of the model-free implied variance (MFIV) underlying the VIX methodology is to use the above relation between the strike and the European option price 
to obtain a model-free measure of market expectation of $\langle\log (S)\rangle_{T}$. It is wellknown that any European style convex payoff is replicated by a static portfolio of the underlying asset, the call and put options; we have

$$
\begin{aligned}
f\left(S_{T}\right)= & f\left(S_{0}\right)+f^{\prime}\left(S_{0}\right)\left(S_{T}-S_{0}\right) \\
& +\int_{\left(0, S_{0}\right]}\left(K-S_{T}\right)_{+} f^{\prime}(\mathrm{d} K)+\int_{\left(S_{0}, \infty\right)}\left(S_{T}-K\right)_{+} f^{\prime}(\mathrm{d} K)
\end{aligned}
$$

for any convex function $f$. This identity is model-free and easily shown by the integration by parts formula of the Stieltjes integral with respect to the right derivative $f^{\prime}$ of $f$. Considering the case $f=-2 \log$, the fair strike of the variance swap is given by

$$
V_{0}=2 \int_{0}^{S_{0}} P_{0}(K) \frac{\mathrm{d} K}{K^{2}}+2 \int_{S_{0}}^{\infty} C_{0}(K) \frac{\mathrm{d} K}{K^{2}},
$$

where $P(K)=P^{1}(K) / S^{0}$ and $C(K)=C^{1}(K) / S^{0}$ with $P^{1}(K)$ and $C^{1}(K)$ referring to respectively the market price processes of the put and call options with strike $K$ and maturity $T$. More generally we can conclude that the fair strike at time $t \in[0, T]$ of a weighted variance swap which has floating leg

$$
\int_{t}^{T} g\left(S_{u}\right) \mathrm{d}\langle\log (S)\rangle_{u}
$$

is given by

$$
V_{t}[g]=\int_{\left(0, S_{t}\right]} P_{t}(K) f_{g}^{\prime}(\mathrm{d} K)+\int_{\left(S_{t}, \infty\right)} C_{t}(K) f_{g}^{\prime}(\mathrm{d} K)
$$

and that the swap contract can be hedged in a model-free manner. For more practical and historical details of the variance swap and the CBOE VIX, see Gatheral [8] and Carr and Lee [1].

As seen so far, provided a market of the put and call options, any European style convex payoff can be replicated by a static portfolio with no knowledge on the dynamics of $S$. Under the continuity assumption on $S$, any weighted variance swap can be replicated by a semi-static portfolio in a model-free manner. Here a semi-static portfolio means a combination of a static portfolio in the option market and a dynamic portfolio in the underlying asset market. Then it 
would be natural to ask how the model-free replication theory can be extended by allowing dynamic rebalancing of replicating portfolios in the option market as in the underlying asset market. In this paper, we give a rigorous formulation of this problem under the assumption that $P(K)$ and $C(K)$ are continuous for all $K>0$. In particular we observe that the swap contract with floating leg $\langle S, V\rangle_{T}$ can be replicated in a model-free manner, where $V$ is the fair strike process of the variance swap, that is, $V_{t}=V_{t}[1]$. Since $V$ is what we call the MFIV, the fair strike of this swap may be interpreted as a model-free measure of market expectation of the leverage effect. We therefore propose to call it the modelfree implied leverage (MFIL). The leverage effect, which refers to a negative relation between an asset price and its volatility movement, is widely observed in empirical studies. Also in calibration of a parametric stochastic volatility model such as the Heston model, the implied leverage parameter usually takes a non-zero value. The negative correlation between the SPX and VIX is actually the major motivation for those who trade the VIX future of the CBOE to do so. It is also known to be the source of the Black-Scholes implied volatility skew and so, the reason why the VIX is sometimes referred as the fear gauge. The MFIL serves as a benchmark of the leverage effect predicted by the market participants. It is as model-free as the MFIV. We may think of it as the fear gauge of the fear gauge in that it measures how much the fear gauge includes negative sentiment of the market participants. The MFIL turns out essentially the same as the slope introduced by Neuberger [10].

The second part of this paper shows two remarkably simple relations between the MFIL and the Black-Scholes implied variance surface. One of them is an extension of Gatheral's elegant formula for the MFIV. The other characterizes the MFIV and MFIL as the coefficients of the leading terms of the asymptotic expansion of the Black-Scholes implied variance. A related property was formally shown by Neuberger [10] but we give more rigorous results. The asymptotic expansion formula is validated in a unified framework which 
incorporates several existing techniques such as the regular perturbation, singular perturbation and small vol-of-vol expansions. Although the framework is non-parametric, we demonstrate some explicit calculations under the Heston model in order to get an intuition of it.

The last part of this paper reports some empirical evidence from the timeseries of the MFIV and MFIL of the Nikkei stock average. The purpose of this empirical study is to confirm the MFIL includes some information which is not captured by the MFIV. More complete empirical analyses are left for future research. See also Neuberger [10]. We observe that the MFIL reflected the fear of the market participants for the effect of a huge earthquake and tsunami which hit Japan on March 11, 2011. The joint dynamics of the MFIV and MFIL clearly distinguished the disaster and the financial crisis on 2008, the two different kinds of turmoil. Further we demonstrate a parametric estimation using YUIMA, an R package for estimations and simulations of stochastic differential equations. It indicates the existence of independent factors which drive the time-series of the MFIV and MFIL.

\section{Tradable assets and attainable processes}

\subsection{Definitions and assumptions}

Here we give a rigorous setup of the model-free replication. Let $(\Omega, \mathcal{F})$ be a measurable space and $T>0$ be a fixed constant. We suppose that three measurable maps $S: \Omega \times[0, T] \rightarrow(0, \infty), P(\cdot): \Omega \times[0, T] \times(0, \infty) \rightarrow[0, \infty)$, $C(\cdot): \Omega \times[0, T] \times(0, \infty) \rightarrow[0, \infty)$ are given and satisfy

1. for each $\omega \in \Omega$ and $K \in(0, \infty)$,

$$
\begin{aligned}
& {[0, T] \ni t \mapsto S_{t}(\omega) \in(0, \infty),} \\
& {[0, T] \ni t \mapsto P_{t}(K)(\omega) \in[0, \infty)}
\end{aligned}
$$

are continuous and $P_{T}(K)(\omega)=\left(K-S_{T}(\omega)\right)_{+}$, 
2. for each $\omega \in \Omega$ and $t \in[0, T]$,

$$
(0, \infty) \ni K \mapsto P_{t}(K)(\omega) \in[0, \infty)
$$

is convex and

$$
\lim _{K \rightarrow 0} P_{t}(K)(\omega)=\lim _{K \rightarrow 0} P_{t}^{\prime}(K)(\omega)=0, \quad \lim _{K \rightarrow \infty} P_{t}^{\prime}(K)(\omega)=1,
$$

where $P^{\prime}$ stands for the right derivative of $P$ with respect to $K$,

3. for each $\omega \in \Omega, K \in(0, \infty)$ and $t \in[0, T]$,

$$
C_{t}(K)(\omega)=P_{t}(K)(\omega)+S_{t}(\omega)-K
$$

Of course $P(K)$ and $S$ stand for the forward price processes of the put option and the underlying asset respectively. The second condition excludes static arbitrage opportunities at any time $t \in[0, T]$ with the put options. Since $P^{\prime}(\cdot)(\omega)$ is non-decreasing and right continuous for each $\omega \in \Omega$, it is a distribution function on $(0, \infty)$ and an integration by parts gives that

$$
P_{t}(K)=\int_{(0, K]} P_{t}^{\prime}(s) \mathrm{d} s=\int_{(0, \infty)}(K-s)_{+} P_{t}^{\prime}(\mathrm{d} s),
$$

where $P_{t}^{\prime}(\mathrm{d} s)$ stands for the Stieltjes measure with respect to $P_{t}^{\prime}$. The last condition is the put-call parity that makes $C(K)$ be the forward price process of the call option. One of easy but important consequences from the above is that

$$
0 \leq P_{t}(K)(\omega) \leq K, \quad 0 \leq C_{t}(K)(\omega) \leq S_{t}(\omega)
$$

for any $t \in[0, T], K \in(0, \infty)$ and $\omega \in \Omega$.

For a given convex function $f$ and $t \in[0, T]$, put

$$
Q_{t}[f]=f\left(S_{0}\right)+f^{\prime}\left(S_{0}\right)\left(S_{t}-S_{0}\right)+\int_{\left(0, S_{0}\right]} P_{t}(K) f^{\prime}(\mathrm{d} K)+\int_{\left(S_{0}, \infty\right)} C_{t}(K) f^{\prime}(\mathrm{d} K),
$$

where $f^{\prime}(\mathrm{d} K)$ stands for the Stieltjes measure with respect to the right derivative $f^{\prime}$ of $f$. By (2), $Q_{t}[f]$ is the forward value at time $t$ of the replicating portfolio 
initiated at time 0 of the European option with payoff $f\left(S_{T}\right)$ and maturity $T$. To exclude an arbitrage opportunity, $Q_{t}[f]$ must coincide with the forward replication cost at time $t$ of the same option for any $t \in[0, T]$;

$$
\begin{aligned}
Q_{t}[f] & =f\left(S_{t}\right)+\int_{\left(0, S_{t}\right]} P_{t}(K) f^{\prime}(\mathrm{d} K)+\int_{\left(S_{t}, \infty\right)} C_{t}(K) f^{\prime}(\mathrm{d} K) \\
& =f\left(S_{t}\right)+\int_{(0, \infty)} \min \left\{P_{t}(K), C_{t}(K)\right\} f^{\prime}(\mathrm{d} K) .
\end{aligned}
$$

The first identity actually follows from the put-call parity using integration by parts for any convex function $f$ and $t \in[0, T]$. The second identity is a simple consequence from the put-call parity. Though $Q[f]=\left\{Q_{t}[f]\right\}$ is well-defined for any convex function $f$, it may be infinite in general.

Definition 2.1 We say $Q[f]$ is a tradable asset if

1. $Q_{t}[f](\omega)$ is finite for any $t \in[0, T], \omega \in \Omega$, and

2. $[0, T] \ni t \mapsto Q_{t}[f](\omega) \in \mathbb{R}$ is continuous for any $\omega \in \Omega$.

By definition, $S, P(K)$ and $C(K)$ are tradable assets for any $K \in(0, \infty)$. More generally we have the following assertion.

Lemma 2.2 If the Stieltjes measure $f^{\prime}$ is finite, then $Q[f]$ is a tradable asset.

Proof: The finiteness of $Q[f]$ follows from (3) and the finiteness of $f^{\prime}$. The families $\left\{P(K) ; K \leq S_{0}\right\}$ and $\left\{C(K) ; K \geq S_{0}\right\}$ are equi-continuous in $t \in[0, T]$ since $\left\{P_{t}(\cdot)\right\}$ and $\left\{C_{t}(\cdot)\right\}$ are families of continuous monotone functions and for each $K \in(0, \infty), P(K)$ and $C(K)$ are continuous in $t$. The continuity of $Q[f]$ then follows from the equi-continuity of the integrands since $f^{\prime}$ is a finite measure. IIII

Let $\left\{\mathcal{F}_{t}\right\}$ be the filtration defined by

$$
\mathcal{F}_{t}=\sigma\left(S_{u}, P_{u}(K) ; u \leq t, K \in(0, \infty)\right) .
$$

Let $\left\{\mathcal{G}_{t}\right\}$ be a filtration of $(\Omega, \mathcal{F})$ with $\mathcal{F}_{t} \subset \mathcal{G}_{t} \subset \mathcal{F}$ for all $t \in[0, T]$. Let $Q$ be the set of probability measures $\mathbb{Q}$ on $(\Omega, \mathcal{F})$ such that $S, P(K), K \in(0, \infty)$ are 
$\left\{\mathcal{G}_{t}^{\mathbb{Q}}\right\}$-local martingales under $\mathbb{Q}$, where $\mathcal{G}_{t}^{\mathbb{Q}}=\cap_{u>t}\left(\mathcal{G}_{u} \vee \mathcal{N}^{\mathbb{Q}}\right)$ and $\mathcal{N}^{\mathbb{Q}}$ is the null sets of the probability space $(\Omega, \mathcal{F}, \mathbb{Q})$. We suppose that $Q$ is not empty. Note that as in the construction of the Wiener space, we can use the canonical path space

$$
\Omega_{0}=\left\{\omega=\left(\omega_{1}, \omega_{2}\right) ;(t, K) \mapsto\left(\omega_{1}(t), \omega_{2}(t, K)\right) ; \text { continuous }\right\}
$$

equipped with the compact uniform topology to construct $(\Omega, \mathcal{F})$ as its Borel subspace with the correspondence

$$
S_{t}(\omega)=\omega_{1}(t), \quad P_{t}(K)(\omega)=\omega_{2}(t, K)
$$

and prove in fact that $Q$ is not empty in this case by noticing that $\Omega$ includes at least all the Black-Scholes scenarios.

Lemma 2.3 Let $f$ be a convex function and $\mathbb{Q} \in Q$. If $Q[f]$ is a tradable asset, then it is a $\left\{\mathcal{G}_{t}^{\mathbb{Q}}\right\}$-local martingale under $\mathbb{Q}$.

Proof: By monotonicity we have $P_{t}(K) \leq P_{t}\left(S_{0}\right)$ for $K \leq S_{0}$ and $C_{t}(K) \leq C_{t}\left(S_{0}\right)$ for $K \geq S_{0}$ for all $t \in[0, T]$. Let

$$
\tau_{n}=\inf \left\{t>0 ; S_{t} \vee P_{t}\left(S_{0}\right) \vee C_{t}\left(S_{0}\right) \vee\left|Q_{t}[f]\right| \geq n\right\}
$$

for $n \in \mathbb{N}$. Then $S_{\cdot \wedge \tau_{n}}, P \cdot \wedge \tau_{n}\left(K_{P}\right)$ and $C_{\cdot \wedge \tau_{n}}\left(K_{C}\right)$ are uniformly integrable and so, $\left\{\mathcal{G}_{t}^{\mathrm{Q}}\right\}$-martingales for all $K_{P} \leq S_{0}$ and $K_{C} \geq S_{0}$. Let $0 \leq u \leq t \leq T$ and $X$ be an arbitrary bounded $\mathcal{G}_{u}^{\mathrm{Q}}$-measurable random variable. Then by the Fubini-Tonelli theorem,

$$
\begin{aligned}
\mathbb{E}_{\mathbb{Q}}\left[X Q_{t \wedge \tau_{n}}[f]\right]= & \mathbb{E}_{\mathbb{Q}}\left[X f\left(S_{0}\right)\right]+\mathbb{E}_{\mathbb{Q}}\left[X f^{\prime}\left(S_{0}\right)\left(S_{t \wedge \tau_{n}}-S_{0}\right)\right] \\
& +\int_{\left(0, S_{0}\right]} \mathbb{E}_{\mathbb{Q}}\left[X P_{t \wedge \tau_{n}}(K)\right] f^{\prime}(\mathrm{d} K)+\int_{\left(S_{0}, \infty\right)} \mathbb{E}_{\mathbb{Q}}\left[X C_{t \wedge \tau_{n}}(K)\right] f^{\prime}(\mathrm{d} K) \\
= & \mathbb{E}_{\mathbb{Q}}\left[X f\left(S_{0}\right)\right]+\mathbb{E}_{\mathbb{Q}}\left[X f^{\prime}\left(S_{0}\right)\left(S_{u \wedge \tau_{n}}-S_{0}\right)\right] \\
& +\int_{\left(0, S_{0}\right]} \mathbb{E}_{\mathbb{Q}}\left[X P_{u \wedge \tau_{n}}(K)\right] f^{\prime}(\mathrm{d} K)+\int_{\left(S_{0}, \infty\right)} \mathbb{E}_{\mathbb{Q}}\left[X C_{u \wedge \tau_{n}}(K)\right] f^{\prime}(\mathrm{d} K) \\
= & \mathbb{E}_{\mathbb{Q}}\left[X Q_{u \wedge \tau_{n}}[f]\right] .
\end{aligned}
$$


Since $\tau_{n} \rightarrow \infty$ as $n \rightarrow \infty, Q[f]$ is a $\mathbb{Q}$-local martingale.

We regard the tradable assets as hedging instruments and define the class of stochastic processes which can be replicated in a model-free manner.

Definition 2.4 We say a $\left\{\mathcal{G}_{t}\right\}$-adapted process $X: \Omega \times[0, T] \rightarrow \mathbb{R}$ is attainable, or an attainable process, if it is continuous $\mathbb{Q}$-almost surely for all $\mathbb{Q} \in Q$ and if there exists a finite set of convex functions $f_{1}, \ldots f_{n}, n \in \mathbb{N}$ such that $Q\left[f_{j}\right]$ is a tradable asset for each $j$ and that there exists a $\left\{\mathcal{G}_{t}\right\}$-progressively measurable process $\left(H^{1}, \ldots H^{n}\right)$ satisfying

$$
\sum_{j=1}^{n} \int_{0}^{T}\left|H_{s}^{j}\right|^{2} \mathrm{~d}\left\langle Q\left[f_{j}\right]\right\rangle_{s}<\infty, \quad X_{t}=X_{0}+\sum_{j=1}^{n} \int_{0}^{t} H_{s}^{j} \mathrm{~d} Q_{s}\left[f_{j}\right]
$$

for all $t \in[0, T], \mathbb{Q}$-almost surely for all $\mathbb{Q} \in \mathbf{Q}$.

Definition 2.5 We say a $\left\{\mathcal{G}_{t}\right\}$-adapted process $X: \Omega \times[0, T] \rightarrow \mathbb{R}$ is semi-attainable, or a semi-attainable process, if it is the sum of an attainable process and $a\left\{\mathcal{G}_{t}\right\}$-adapted process whose path is continuous and of finite variation $\mathbb{Q}$-almost surely for all $\mathbb{Q} \in \mathbf{Q}$.

If $X$ is an attainable process, then the derivative with payoff $X_{t}$ with maturity $t \in[0, T]$ can be hedged with forward replication cost at time $s \in[0, t]$ being $X_{s}$. The hedging strategy $\left(H^{1}, \ldots, H^{n}\right)$ does not depend on $\mathbb{Q}$. This interpretation assumes the existence of a local martingale measure which is equivalent to the law $\mathbb{P}$ on $(\Omega, \mathcal{F})$ that the underlying asset price $S$ and the put and call option prices $P(K), C(K), K \in(0, \infty)$ actually follow. This assumption is equivalent to the No Free Lunch condition:

$$
\begin{aligned}
& \overline{M-L_{+}} \cap L_{+}=\{0\}, \\
& L_{+}=\left\{X \in L^{\infty}(\Omega, \mathcal{F}, \mathbb{P}) \mid X \geq 0\right\},
\end{aligned}
$$

where $M$ is the subspace of $L^{\infty}(\Omega, \mathcal{F}, \mathbb{P})$ generated by the elements of the form

$$
H_{\tau \wedge \sigma}\left(S_{\tau}-S_{\tau \wedge \sigma}\right), F_{\tau \wedge \sigma}\left(P_{\tau}(K)-P_{\tau \wedge \sigma}(K)\right)
$$

with $K \in(0, \infty)$, finite $\left\{\mathcal{G}_{t}^{\mathbb{P}}\right\}$-stopping times $\tau$ and $\sigma$ such that $\sup _{t \geq 0} S_{t \wedge \tau}$ and $\sup _{t \geq 0} P_{t \wedge \tau}(K)$ are bounded, and $\mathcal{G}_{\tau \wedge \sigma}^{\mathbb{P}}$-measurable bounded random variables 
$H_{\tau \wedge \sigma}$ and $F_{\tau \wedge \sigma}$. Here the closure $\overline{M-L_{+}}$of $M-L_{+}$is in $\sigma\left(L^{\infty}, L^{1}\right)$, that is, the weak * topology. This equivalence follows from Theorem 5.2.3 of Delbaen and Schachermayer [3]. The point is that we do not specify $\mathbb{P}$ but still can replicate $X_{t}$ by a model-free hedging strategy, $\mathbb{P}$-almost surely under the No Free Lunch condition.

\subsection{Examples of attainable process}

Here we give examples of attainable process. By definition, every tradable asset is an attainable process. In particular $S, P(K)$ and $C(K)$ are attainable. Obviously the sum of two attainable processes is attainable. Let $X$ be a semi-attainable process and $H$ be a $\left\{\mathcal{G}_{t}\right\}$-adapted process whose path is cadlag $\mathbb{Q}$-almost surely for all $\mathbb{Q} \in Q$. Then we can define a $\left\{\mathcal{G}_{t}\right\}$-adapted process $H \cdot X$ as

$$
H \cdot X_{t}(\omega):=\left\{\begin{array}{l}
0 \quad \text { if } \liminf _{n \rightarrow \infty} \sum_{j=0}^{\infty} H_{\tau_{j}^{n}}(\omega)\left(X_{\tau_{j+1}^{n} \wedge t}(\omega)-X_{\tau_{j}^{n} \wedge t}(\omega)\right) \notin \mathbb{R}, \\
\liminf _{n \rightarrow \infty} \sum_{j=0}^{\infty} H_{\tau_{j}^{n}}(\omega)\left(X_{\tau_{j+1}^{n} \wedge}(\omega)-X_{\tau_{j}^{n} \wedge t}(\omega)\right) \quad \text { otherwise, }
\end{array}\right.
$$

where

$$
\tau_{0}^{n}=0, \quad \tau_{j+1}^{n}=\inf \left\{t>\tau_{j}^{n} ;\left|H_{t}-H_{\tau_{j}^{n}}\right| \geq 2^{-n}\right\} .
$$

For any $\mathbb{Q} \in Q$, the limit exists and gives a continuous version of the stochastic integral of $H$ with respect to $X, \mathbb{Q}$-almost surely. See Karandikar [9] for the detail. If $X$ is attainable, then the defined map $H \cdot X$ is clearly an example of attainable process. Moreover we can define the quadratic covariation $\langle X, Y\rangle$ of two semi-attainable processes $X$ and $Y$ as a measurable map on $\Omega \times[0, T]$ :

$$
\langle X, Y\rangle_{t}(\omega):=X_{t}(\omega) Y_{t}(\omega)-X_{0}(\omega) Y_{0}(\omega)-X \cdot Y_{t}(\omega)-Y \cdot X_{t}(\omega)
$$

By this definition, $X Y-\langle X, Y\rangle$ is attainable if $X$ and $Y$ are attainable. Put $\langle X\rangle:=\langle X, X\rangle$ for a semi-attainable process $X$. This is again semi-attainable.

Proposition 2.6 Let $X$ be an attainable process and $f$ be a differentiable function with absolutely continuous derivative $f^{\prime}$ defined on a domain which includes the image $\left\{X_{t} \mid t \in[0, T]\right\}, \mathbb{Q}$-almost surely for all $\mathbb{Q} \in Q$. Then $f(X)$ is semi-attainable and

$$
f(X)-\frac{1}{2} \int_{0} f^{\prime \prime}\left(X_{t}\right) \mathrm{d}\langle X\rangle_{t},\langle f(X)\rangle-\int_{0}\left|f^{\prime}\left(X_{t}\right)\right|^{2} \mathrm{~d}\langle X\rangle_{t}
$$

are attainable. 
Proof: This follows from the Itô-Tanaka formula.

Proposition 2.7 Let $X$ be an attainable process. Let $h_{j}, j \in \mathbb{Z}_{+}$, be the $j$-th Hermite polynomial defined by

$$
\exp \left\{t x-\frac{t^{2}}{2}\right\}=\sum_{j=0}^{\infty} h_{j}(x) \frac{t^{j}}{j !}
$$

or equivalently,

$$
h_{j}(x)=\int_{\mathbb{R}}(x+i z)^{j} \Phi(\mathrm{d} z),
$$

where $i=\sqrt{-1}$ and $\Phi$ is the standard normal distribution function. Then

$$
\begin{aligned}
& e(X):=\exp \left\{X-\frac{1}{2}\langle X\rangle\right\}, \\
& H_{j}(X):=\langle X\rangle^{j / 2} h_{j}\left(\langle X\rangle^{-1 / 2} X\right)
\end{aligned}
$$

are attainable.

Proof: This is because $\varphi(x, t):=\exp (x-t / 2), \varphi_{j}(x, t):=t^{j / 2} h_{j}\left(t^{-1 / 2} x\right)$ satisfy

$$
\frac{\partial \varphi}{\partial t}+\frac{1}{2} \frac{\partial^{2} \varphi}{\partial x^{2}}=0, \frac{\partial \varphi_{j}}{\partial t}+\frac{1}{2} \frac{\partial^{2} \varphi_{j}}{\partial x^{2}}=0
$$

for any $j \in \mathbb{Z}_{+}$. Then apply Itô's formula to have the result.

Corollary 2.8 Attainable are

$$
\begin{aligned}
& \log (S)+\frac{1}{2}\langle\log (S)\rangle \\
& S \log (S)+\frac{1}{2} S\langle\log (S)\rangle-\langle S, \log (S)\rangle \\
& \log (S)\langle\log (S)\rangle+\frac{1}{4}\langle\log (S)\rangle^{2}+(\log (S))^{2}-\langle\log (S)\rangle
\end{aligned}
$$

\subsection{Volatility derivatives}

As seen in Introduction, any weighted variance swap is hedged by a model-free strategy. This fact is described as follows in terms of attainable process.

Proposition 2.9 Let $g$ be a locally integrable non-negative function and $f_{g}$ be defined by (1). Put $V[g]=\left\{V_{t}[g]\right\}, V_{t}[g]=Q_{t}\left[f_{g}\right]-f_{g}\left(S_{t}\right)$. If $Q\left[f_{g}\right]$ is a tradable asset, then

$$
V[g]+\int_{0} g\left(S_{t}\right) \mathrm{d}\langle\log (S)\rangle_{t}
$$


is attainable and

$$
V[g]=\int_{(0, \infty)} \min \{P(K), C(K)\} f_{g}^{\prime}(\mathrm{d} K), \quad V_{T}[g]=0 .
$$

Proof: This is a combination of Proposition 2.6, the fact that a tradable asset is attainable, and the identity (4).

The fair strike at time $s \in[0, T]$ of the weighted variance swap with maturity $T$ and floating leg

$$
\int_{s}^{T} g\left(S_{t}\right) \mathrm{d}\langle\log (S)\rangle_{t}=V_{T}[g]+\int_{0}^{T} g\left(S_{t}\right) \mathrm{d}\langle\log (S)\rangle_{t}-\int_{0}^{s} g\left(S_{t}\right) \mathrm{d}\langle\log (S)\rangle_{t}
$$

is therefore given by $V_{s}[g]$. Put $V=\left\{V_{t}\right\}, V_{t}=V_{t}[1]$, which represents the fair strike of the variance swap. Combining Propositions 2.7 and 2.9, we obtain the following corollary.

Corollary 2.10 If $V$ is finite and continuous, then

$$
V+\langle\log (S)\rangle, \quad(V+\langle\log (S)\rangle)^{2}-\langle V\rangle, \quad S(V+\langle\log (S)\rangle)-\langle S, V\rangle
$$

are attainable.

In particular we find that the fair strike at time 0 of the swap contract with maturity $T$ and floating leg $\langle\log (S)\rangle_{T}^{2}-\langle V\rangle_{T}$ is $V_{0}^{2}$. Since $V_{0}$ is the expected value of $\langle\log (S)\rangle_{T}$ under the pricing measure $Q$, we reach a surprisingly simple conclusion that the variance of $\langle\log (S)\rangle_{T}$ under $Q$ coincides with the expected value of $\langle V\rangle_{T}$ under $Q$. Similarly the covariance between $S_{T}$ and $\langle\log (S)\rangle_{T}$ under $Q$ coincides with the expectation of $\langle S, V\rangle_{T}$ under $Q$. The quantity $V$ has been paid much attention in industry as a model-free measure of the volatility of $S$. The CBOE is dealing with the future and options of the VIX that represents $100 \sqrt{V_{0} / T}$ with $T=30$ days.

Besides the aspect of $V$ as a descriptive statistics of the option data, the importance of trading the variance swap may be explained as follows. For a 
given Borel function $f:(0, \infty) \rightarrow \mathbb{R}$ of polynomial growth, define $p^{f}:(0, \infty) \times$ $[0, \infty) \rightarrow \mathbb{R}$ as

$$
p^{f}(s, t)=\int_{\mathbb{R}} f(s \exp \{\sqrt{t} z-t / 2\}) \Phi(\mathrm{d} z),
$$

where $\Phi$ is the standard normal distribution function. A direct calculation gives the partial differential equation

$$
\frac{\partial p^{f}}{\partial t}=\frac{1}{2} s^{2} \frac{\partial^{2} p^{f}}{\partial s^{2}}
$$

with boundary condition $p^{f}(s, 0)=f(s)$ for all $s \in(0, \infty)$. One can notice that $S_{t}^{0} p^{f}\left(S_{t}, \sigma^{2}(T-t)\right)$ is the Black-Scholes price at time $t$ with volatility $\sigma$ of the European option with payoff $f$ and maturity $T$. Now taking $f=-\log$, we have

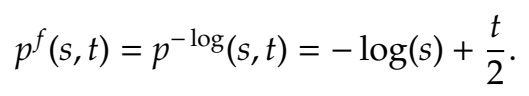

By definition, for all $t \in[0, T]$,

$$
p^{-\log }\left(S_{t}, V_{t}\right)=Q_{t}[-\log ]
$$

This identity allows us to interpret $\sqrt{V_{t} /(T-t)}$ as the Black-Scholes implied volatility corresponding to the payoff $f=-\log$. The speciality of this payoff is that

$$
\frac{\partial p^{f}}{\partial t}=\frac{1}{2} s^{2} \frac{\partial^{2} p^{f}}{\partial s^{2}}=\frac{1}{2}, \frac{\partial^{2} p^{f}}{\partial s \partial s}=\frac{\partial^{2} p^{f}}{\partial t^{2}}=0 .
$$

This "constant Vega" property is attractive to financial practitioners who want to control their exposure to the volatility risk. As seen in Introduction, trading the variance swap is essentially equivalent to trading this log contract.

Another payoff enjoying a special property is $f(s)=s \log (s)$. For this, we have that $p^{f}(s, t)=s \log (s)+s t / 2$, so that

$$
\frac{\partial p^{f}}{\partial t}=\frac{1}{2} s^{2} \frac{\partial^{2} p^{f}}{\partial s^{2}}=\frac{s}{2}, \frac{\partial^{2} p^{f}}{\partial s \partial s}=\frac{1}{2}, \frac{\partial^{2} p^{f}}{\partial t^{2}}=0 .
$$

Let $g(s)=s$ be the identity map and

$$
G_{t}=\frac{1}{S_{t}} V_{t}[g]=\frac{2}{S_{t}} \int_{0}^{S_{t}} \frac{P_{t}(K)}{K} \mathrm{~d} K+\frac{2}{S_{t}} \int_{S_{t}}^{\infty} \frac{C_{t}(K)}{K} \mathrm{~d} K .
$$


Then

$$
p^{f}\left(S_{t}, G_{t}\right)=Q_{t}[f] .
$$

This means that $\sqrt{G_{t} /(T-t)}$ is the Black-Scholes implied volatility corresponding to the payoff $f(s)=s \log (s)$. Under the Black-Scholes model with volatility $\sigma$, we have that $G_{t}=V_{t}=\sigma^{2}(T-t)$ for all $t \in[0, T]$. If the Black-Scholes implied volatility smile is symmetric at time $t$, then we still have $G_{t}=V_{t}$. See Carr and Lee [2] for the detail of this symmetry.

Proposition 2.11 If $Q[f]$ is a tradable asset for $f(s)=s \log (s)$, or equivalently, $G$ is finite and continuous, then

$$
\langle\log (S)\rangle+\langle\log (S), G\rangle+G
$$

is attainable. If, in addition, $V$ is finite and continuous, then

$$
\langle\log (S), G\rangle+G-V
$$

is attainable.

Proof: Notice that $S$ and $G$ are semi-martingales under $\mathbb{Q}$ for any $\mathbb{Q} \in \mathbb{Q}$. By Itô's formula,

$$
\frac{\mathrm{d} Q_{t}[f]}{S_{t}}=\frac{\partial p^{f}}{\partial s}\left(S_{t}, G_{t}\right) \frac{\mathrm{d} S_{t}}{S_{t}}+\frac{1}{2}\left[\mathrm{~d}\langle\log (S)\rangle_{t}+\mathrm{d} G_{t}+\mathrm{d}\langle\log (S), G\rangle_{t}\right]
$$

under $\mathbb{Q}$ for any $\mathbb{Q} \in Q$, which gives the result. If $V$ is finite and continuous, we can combine this with Corollary 2.10.

IIII

In particular we find that the fair strike at time 0 of the swap contract with floating $\operatorname{leg}\langle\log (S), G\rangle_{T}$ is $G_{0}-V_{0}$. This fact has been already found by Neuberger [10]. By definition, $G_{t}$ is the fair strike at time $t$ of the Gamma swap with maturity $T$, that is, the swap with floating leg

$$
\int_{t}^{T} \frac{S_{u}}{S_{t}} \mathrm{~d}\langle\log (S)\rangle_{u}
$$

Due to the weight $S_{u} / S_{t}$, this swap contract is more attractive than the variance swap to those who expect some correlation structure between $S$ and $\langle\log (S)\rangle$. Itô's formula and Corollary 2.10 give the following corollary. 
Corollary 2.12 If $G$ is finite and continuous, then

$$
S G+\int_{0} S_{u} \mathrm{~d}\langle\log (S)\rangle_{u}, \quad S G+S\langle\log (S)\rangle
$$

are attainable. If, in addition, $V$ is finite and continuous, then

$$
S(G-V)+\langle S, V\rangle
$$

is attainable.

We come back to these attainable processes in the next section. So far we consider $f(s)=-\log (s)$ and $f(s)=s \log (s)$ to obtain simple structures of the higher order derivatives of $p^{f}$ which yield rather neat attainable processes. Next we treat the power payoff $f(s)=2 s^{\alpha} /(\alpha(\alpha-1)), \alpha \in \mathbb{R} \backslash\{0,1\}$. A straightforward calculation gives that

$$
p^{f}(s, t)=\frac{2}{\alpha(\alpha-1)} s^{\alpha} \exp \left\{\frac{1}{2} \alpha(\alpha-1) t\right\}
$$

and

$$
\frac{\partial p^{f}}{\partial t}=\frac{1}{2} s^{2} \frac{\partial^{2} p^{f}}{\partial s^{2}}=\frac{1}{2} \alpha(\alpha-1) p^{f}=\frac{s}{\alpha} \frac{\partial^{2} p^{f}}{\partial s \partial t}=\frac{2}{\alpha(\alpha-1)} \frac{\partial^{2} p^{f}}{\partial t^{2}} .
$$

Define $\Sigma(\alpha)=\left\{\Sigma_{t}(\alpha)\right\}$ by the equation

$$
Q_{t}[f]=p^{f}\left(S_{t}, \Sigma_{t}(\alpha)\right)
$$

or equivalently, putting $g_{\alpha}(s)=s^{\alpha}$,

$$
V_{t}\left[g_{\alpha}\right]=\frac{2}{\alpha(\alpha-1)} S^{\alpha}\left(\exp \left\{\frac{1}{2} \alpha(\alpha-1) \Sigma_{t}(\alpha)\right\}-1\right) .
$$

The first identity means that $\sqrt{\sum_{t}(\alpha) /(T-t)}$ is the Black-Scholes implied volatility at time $t$ corresponding to the payoff $f(s)=2 s^{\alpha} /(\alpha(\alpha-1))$.

Proposition 2.13 If $Q[f]$ is a tradable asset, or equivalently, $\Sigma(\alpha)$ is finite and continuous, then

$$
\langle\log (S)\rangle+\Sigma(\alpha)+\alpha\langle\log (S), \Sigma(\alpha)\rangle+\frac{\alpha(\alpha-1)}{4}\langle\Sigma(\alpha)\rangle
$$

is attainable. 
Proof: As in the proof of the previous proposition, Itô's formula gives that

$$
\begin{aligned}
& \langle\log (S)\rangle_{t}+\Sigma_{t}(\alpha)+\alpha\langle\log (S), \Sigma(\alpha)\rangle_{t}+\frac{\alpha(\alpha-1)}{4}\langle\Sigma(\alpha)\rangle_{t} \\
& =\frac{2}{\alpha(\alpha-1)} \int_{0}^{t} \frac{\mathrm{d} Q_{u}[f]}{Q_{u}[f]}-\frac{2}{\alpha-1} \int_{0}^{t} \frac{\mathrm{d} S_{u}}{S_{u}}
\end{aligned}
$$

for all $t \in[0, T]$.

Corollary 2.14 If $V\left[g_{\alpha}\right]$ is finite and continuous, then

$$
V\left[g_{\alpha}\right]+\int_{0} S_{u}^{\alpha} \mathrm{d}\langle\log (S)\rangle_{u}, \quad S V\left[g_{\alpha}\right]+\int_{0} S_{u}^{\alpha+1} \mathrm{~d}\langle\log (S)\rangle_{u}-\langle S, V(\alpha)\rangle
$$

are attainable. If $V\left[g_{\alpha+1}\right]$ is finite and continuous in addition, then

$$
V\left[g_{\alpha+1}\right]-S V\left[g_{\alpha}\right]+\left\langle S, V\left[g_{\alpha}\right]\right\rangle
$$

is attainable.

Remark that the cases $\alpha=0$ and $\alpha=1$ formally correspond to $V$ and $G$ respectively.

\section{Implied Leverage}

\subsection{Model-free measure of the leverage effect}

Corollary 2.12 implies that the swap contract with maturity $T$ and floating leg $\langle S, V\rangle_{T}$ is replicated with the fair strike at time 0 being $S_{0}\left(G_{0}-V_{0}\right)$ provided that $G$ and $V$ are finite and continuous. Since $V$ is regarded as the modelfree implied variance (MFIV) of $S$, the quadratic covariation $\langle S, V\rangle_{T}$ may be interpreted as the realized leverage effect of $S$. The fair strike $S_{0}\left(G_{0}-V_{0}\right)$ is the expectation of $\langle S, V\rangle_{T}$ under the pricing measure $Q$. Dividing it by $S_{0} V_{0}$, we put $L_{0}:=G_{0} / V_{0}-1$. By Proposition 2.11 and Corollaries 2.10 and 2.12, $L_{0}$ is the the expectation under $Q$ of the following quantities:

$$
\frac{1}{S_{0} V_{0}}\langle S, V\rangle_{T}, \frac{1}{V_{0}}\langle\log (S), G\rangle_{T},\left(\frac{S_{T}}{S_{0}}-1\right) \frac{\langle\log (S)\rangle_{T}}{V_{0}} .
$$

In particular it coincides with the covariance of $S_{T} / S_{0}$ and $\langle\log (S)\rangle_{T} / V_{0}$ under $Q$. Since $G_{0}$ and $V_{0}$ are uniquely determined by the market prices of the put 
and call options at time 0 without specifying any model, we regard $L_{0}$ as a model-free measure of the leverage effect implied by the option market data. This is exactly in the same manner as considering $V_{0}$ to be a model-free measure or the market's expectation of the volatility of $S$. Motivated by this, we propose to call $L_{0}$ the model-free implied leverage (MFIL). Neuberger [10] found that $G_{0}-V_{0}=V_{0} L_{0}$ is the model-free expectation of $\langle\log (S), G\rangle_{T}$ and called it the slope. Our new approach enabled us to show in particular that $\langle S, V\rangle_{T}$, that is a more natural quantity as a measure of realized leverage effect, can be replicated in a model-free manner as well. The reason why we consider $L_{0}$ instead of $G_{0}-V_{0}$ is that, as we will see, the former naturally arises as the leading term of the Black-Scholes implied variance skew. A related property was formally shown by Neuberger [10], which was the reason why $G_{0}-V_{0}$ was called the slope.

\subsection{Representation}

Here we give a representation formula of the MFIL in terms of the Black-Scholes implied variance. This is an application of Fukasawa [6] extending Gatheral's formula of the MFIV given in Gatheral [8]. The Black-Scholes implied variance $\Sigma_{\mathrm{BS}}(k), k \in \mathbb{R}$ at time 0 is defined by

$$
P\left(S_{0} e^{k}\right)=S_{0} Q_{\mathrm{BS}}\left(k, \Sigma_{\mathrm{BS}}(k)\right),
$$

where $k$ represents the log-moneyness of strike price, and

$$
\begin{aligned}
& Q_{\mathrm{BS}}(k, \Sigma)=e^{k} \Phi\left(-d_{2}(k, \Sigma)\right)-\Phi\left(-d_{1}(k, \Sigma)\right), \\
& d_{2}(k, \Sigma)=\frac{-k-\Sigma / 2}{\sqrt{\Sigma}}, \\
& d_{1}(k, \Sigma)=d_{2}(k, \Sigma)+\sqrt{\Sigma} .
\end{aligned}
$$

By (3), $\Sigma_{\mathrm{BS}}(k)$ is well-defined for each $k \in \mathbb{R}$ because $\Sigma \mapsto Q_{\mathrm{BS}}(k, \Sigma)$ is strictly increasing and $\lim _{\Sigma \rightarrow \infty} Q_{\mathrm{BS}}(k, \Sigma)=e^{k}, \lim _{\Sigma \rightarrow-\infty} Q_{\mathrm{BS}}(k, \Sigma)=0$. The prices in option markets are usually quoted in terms of the Black-Scholes implied volatility, that is, $\sqrt{\sum_{\mathrm{BS}}\left(\log \left(K / S_{0}\right)\right) / T}$ for each strike price $K$. The following theorem characterizes $G_{0}$ and $V_{0}$ as weighted averages of the Black-Scholes implied variances. 
Theorem 3.1 Suppose that $P_{0}^{\prime}$ is absolutely continuous in $K$ and that there exists $\epsilon>0$ such that

$$
1-P_{0}^{\prime}(K)=O\left(K^{-1-\epsilon}\right) \text {, as } K \rightarrow \infty
$$

and

$$
P_{0}^{\prime}(K)=O\left(K^{\epsilon}\right) \text {, as } K \rightarrow 0 .
$$

Then, $f_{i}: \mathbb{R} \ni k \mapsto-d_{i}\left(k, \Sigma_{\mathrm{BS}}(k)\right) \in \mathbb{R}, i=1,2$ are homeomorphisms and

$$
G_{0}=\int_{\mathbb{R}} \Sigma_{\mathrm{BS}}\left(g_{1}(k)\right) \Phi(\mathrm{d} k), \quad V_{0}=\int_{\mathbb{R}} \Sigma_{\mathrm{BS}}\left(g_{2}(k)\right) \Phi(\mathrm{d} k),
$$

where $g_{i}, i=1,2$ are the inverse functions of $f_{i}$ respectively.

Proof: This follows from Theorem 4.1 of Fukasawa [6].

The assumptions in the above theorem are mild regularity and integrability conditions on the law of $S_{T}$ under the pricing measure $Q$. The former (the absolute continuity of $P_{0}^{\prime}$ ) is equivalent to say $S_{T}$ admits a density under $Q$ and the latter is equivalent to say there exists $\epsilon>0$ such that $S_{T}^{1+\epsilon}$ and $S_{T}^{-\epsilon}$ are integrable under $Q$. From this theorem it is clear that $L_{0}=G_{0} / V_{0}-1=0$ if the Black-Scholes implied variance $\Sigma_{\mathrm{BS}}(\cdot)$ is symmetric, that is, if $\Sigma_{\mathrm{BS}}(k)=\Sigma_{\mathrm{BS}}(-k)$ for all $k \in \mathbb{R}$. Therefore, $L_{0}$ is a measure of the skew in the Black-Scholes implied volatility surface. As shown in Carr and Lee [2], stochastic volatility models with no leverage effect enjoys this symmetry property in general. This fact supports our interpretation that $L_{0}$ is a measure of the leverage effect.

As illustrated in Fukasawa et al. [7], the above type of representation formula has a computational advantage compared to one written in terms of direct integrals of $\left\{P_{0}(K), C_{0}(K) ; K \in(0, \infty)\right\}$. In brief, the formula is involved only with integrals with respect to the standard normal distribution, which enjoys a nice relationship with polynomial interpolations of the volatility surface. We use this formula in our empirical analysis in the last section. 


\subsection{The Heston model}

The model-free hedging theory would be useful in managing financial risks due to various misspecifications of models especially if the relation between model-free measures and their counterparts under models is well-understood. Here we treat the Heston model to illustrate the relation between the MFIL and the model parameters. Suppose

$$
\begin{aligned}
& \mathrm{d} S_{t}=S_{t} \sqrt{Y_{t}}\left(\rho \mathrm{d} W_{t}^{1}+\sqrt{1-\rho^{2}} \mathrm{~d} W_{t}^{2}\right), \\
& \mathrm{d} Y_{t}=\alpha\left(\theta-Y_{t}\right) \mathrm{d} t+\sigma \sqrt{Y_{t}} \mathrm{~d} W_{t}^{1} .
\end{aligned}
$$

Then, for $u \geq t \geq 0$

$$
y(u):=y(u ; t):=\mathbb{E}\left[Y_{u} \mid \mathcal{F}_{t}\right]=Y_{t}+\mathbb{E}\left[\int_{t}^{u} \alpha\left(\theta-Y_{s}\right) \mathrm{d} s\right],
$$

so

$$
y^{\prime}(u)=\alpha(\theta-y(u)),
$$

which implies

$$
y(u ; t)=\theta+\left(Y_{t}-\theta\right) e^{-\alpha(u-t)} .
$$

Therefore

$$
\begin{aligned}
V_{t} & =\mathbb{E}\left[\langle\log (S)\rangle_{T}-\langle\log (S)\rangle_{t} \mid \mathcal{F}_{t}\right] \\
& =\mathbb{E}\left[\int_{t}^{T} Y_{s} \mathrm{~d} s \mid \mathcal{F}_{t}\right] \\
& =\int_{t}^{T} y(s ; t) \mathrm{d} s \\
& =\theta(T-t)+\left(Y_{t}-\theta\right) \frac{1-e^{-\alpha(T-t)}}{\alpha} .
\end{aligned}
$$

Note that $V$ does not depend on $\rho$ and so has no information on the leverage effect. On the other hand, since

$$
\mathrm{d}(S Y)_{t}=Y_{t} \mathrm{~d} S_{t}+S_{t} \mathrm{~d} Y_{t}+\mathrm{d}\langle S, Y\rangle_{t}
$$

we have for $u \geq t \geq 0$,

$$
\begin{aligned}
x(u):=x(u ; t):= & \frac{1}{S_{t}} \mathbb{E}\left[S_{u} Y_{u} \mid \mathcal{F}_{t}\right] \\
& =Y_{t}+\alpha \theta(u-t)-\alpha \int_{t}^{u} x(s) \mathrm{d} s+\sigma \rho \int_{t}^{u} x(s) \mathrm{d} s,
\end{aligned}
$$


and so,

$$
x^{\prime}(u)=\alpha \theta-(\alpha-\sigma \rho) x(u) .
$$

This implies that

$$
x(u ; t)=\frac{\alpha \theta}{\alpha-\sigma \rho}+\left(Y_{t}-\frac{\alpha \theta}{\alpha-\sigma \rho}\right) e^{-(\alpha-\sigma \rho)(u-t)}
$$

and that

$$
\begin{aligned}
G_{t} & =\frac{1}{S_{t}} \mathbb{E}\left[\int_{t}^{T} S_{u} \mathrm{~d}\langle\log (S)\rangle_{u} \mid \mathcal{F}_{t}\right] \\
& =\frac{1}{S_{t}} \mathbb{E}\left[\int_{t}^{T} S_{u} Y_{u} \mathrm{~d} u \mid \mathcal{F}_{t}\right] \\
& =\int_{t}^{T} x(s ; t) \mathrm{d} s \\
& =\frac{\alpha \theta}{\alpha-\sigma \rho}(T-t)+\left(Y_{t}-\frac{\alpha \theta}{\alpha-\sigma \rho}\right) \frac{1-e^{-(\alpha-\sigma \rho)(T-t)}}{\alpha-\sigma \rho} .
\end{aligned}
$$

Thus we can obtain an explicit expression of $L_{0}=G_{0} / V_{0}-1$. Notice that $G$ coincides with $V$ if $\rho=0$.

We can check directly that

$$
\mathbb{E}\left[\langle\log (S), G\rangle_{T}-\langle\log (S), G\rangle_{t} \mid \mathcal{F}_{t}\right]=\frac{1}{S_{t}} \mathbb{E}\left[\langle S, V\rangle_{T}-\langle S, V\rangle_{t} \mid \mathcal{F}_{t}\right]=G_{t}-V_{t}
$$

as follows. Notice that

$$
\mathbb{E}\left[\langle\log (S), G\rangle_{T}-\langle\log (S), G\rangle_{t} \mid \mathcal{F}_{t}\right]=\sigma \rho \int_{t}^{T} \frac{1-e^{-(\alpha-\sigma \rho)(T-s)}}{\alpha-\sigma \rho} y(s ; t) \mathrm{d} s,
$$

and that

$$
\frac{1}{S_{t}} \mathbb{E}\left[\langle S, V\rangle_{T}-\langle S, V\rangle_{t} \mid \mathcal{F}_{t}\right]=\sigma \rho \int_{t}^{T} \frac{1-e^{-\alpha(T-s)}}{\alpha} x(s ; t) \mathrm{d} s .
$$

Both of them are 0 at $t=T$. Differentiating in $T$ yields

$$
\begin{aligned}
& \frac{\sigma \rho \theta}{\alpha-\sigma \rho}\left(1-e^{-(\alpha-\sigma \rho)(T-t)}\right)+\left(Y_{t}-\theta\right)\left(e^{-(\alpha-\sigma \rho)(T-t)}-e^{-\alpha(T-t)}\right) \\
= & \frac{\sigma \rho \theta}{\alpha-\sigma \rho}\left(1-e^{-\alpha(T-t)}\right)+\left(Y_{t}-\frac{\alpha \theta}{\alpha-\sigma \rho}\right)\left(e^{-(\alpha-\sigma \rho)(T-t)}-e^{-\alpha(T-t)}\right) \\
= & x(T ; t)-y(T ; t)
\end{aligned}
$$


for both, which proves the identity.

To get a simpler expression of $L_{0}$, let us consider the fast mean-reverting region of parameters: $\sigma=\sigma_{0} / \epsilon, \alpha=1 / \epsilon^{2}$ and $\epsilon \rightarrow 0$. Then,

$$
L_{0}=\frac{G_{0}}{V_{0}}-1=\frac{\alpha}{\alpha-\sigma \rho}+O\left(\epsilon^{2}\right)=\sigma_{0} \rho \epsilon+O\left(\epsilon^{2}\right) .
$$

Therefore $L_{0}$ is asymptotically proportional to the leverage parameter $\rho$. This fact again supports our interpretation of $L_{0}$ as a measure of the leverage effect. For the fast mean-reverting stochastic volatility models including the Heston case with $|\rho|<1$, Fukasawa [4] proved the validity of a singular perturbation expansion of the European option prices. The result for the Heston case gives as a corollary the asymptotic expansion of the Black-Scholes implied variance

$$
\Sigma_{\mathrm{BS}}(k)=\theta\left(1+\frac{1}{2} \sigma_{0} \rho \epsilon\right)+\sigma_{0} \rho \epsilon k+O\left(\epsilon^{2}\right)
$$

as $\epsilon \rightarrow 0$ for all $k \in \mathbb{R}$. This means that the Black-Scholes implied variance surface is an affine function of log-moneyness up to $O\left(\epsilon^{2}\right)$. Combining this and our calculation above, we have

$$
\Sigma_{\mathrm{BS}}(k)=V_{0}\left(1+\frac{L_{0}}{2}\right)+L_{0} k+O\left(\epsilon^{2}\right) .
$$

Therefore the MFIV and MFIL coincide with the baseline and the slope of the surface respectively. In the next subsection we observe that this remarkable relation remains valid under a more general perturbation structure.

\subsection{Asymptotic expansion}

Here we consider a sequence of stochastic volatility models which converges to the Black-Scholes model. This extends the asymptotic analysis in the previous subsection. We see that the MFIV and MFIL appear in the leading term of the asymptotic expansion of the Black-Scholes implied variance surface. The result is obtained as an application of Fukasawa [5]. We introduce an artificial variable $n \in \mathbb{N}$ which drives the asymptotics and consider a sequence $\mathbb{Q}^{n} \in Q$. 
We suppose $\left(\Omega, \mathcal{F}, \mathbb{Q}^{n}\right)=\left(\Omega^{\prime} \otimes \mathcal{W}, \mathcal{F}^{\prime} \otimes \mathcal{H}, \mathbb{Q}^{n \prime} \otimes \mu\right)$, where $\left(\Omega^{\prime}, \mathcal{F}^{\prime}, \mathbb{Q}^{n, \prime},\left\{\mathcal{F}_{t}^{\prime}\right\}\right)$ is a filtered probability space and $(\mathcal{W}, \mathcal{H}, \mu)$ is the Wiener space with canonical process $W$. Let $X$ be an $\left\{\mathcal{F}_{t}^{\prime}\right\}$-local martingale under $\mathbb{Q}^{n \prime}$ for any $n \in \mathbb{N}$ and $F$ be an $\left\{\mathcal{F}_{t}^{\prime}\right\}$-adapted process. We suppose that $S$ is of the form

$$
\log \left(S_{t} / S_{0}\right)=M_{t}-\frac{1}{2}\langle M\rangle_{t}, M_{t}:=X_{t}+\int_{0}^{t} F_{s} \mathrm{~d} W_{s} .
$$

under $\mathbb{Q}^{n}$ for all $n \in \mathbb{N}$. This is an abstract representation of $S$ and up to this point there is essentially no restriction on $S$ other than its continuity. Regarding $\mathbb{Q}^{n}$ as the pricing measure, the MFIV is given as $V^{n}:=\mathbb{E}_{\mathbb{Q}^{n}}\left[\langle M\rangle_{T}\right]$. The idea of the following asymptotic analysis is to consider the pricing measure $Q$ to be in some sense close to a probability measure $\mathbb{Q}^{\mathrm{BS}}$ under which $S_{T}$ follows a lognormal distribution. This generalizes analyses for various perturbation models around the Black-Scholes model. We embed $Q$ in the sequence $\mathbb{Q}^{n}$ and let $\mathbb{Q}^{n}$ converge to $\mathbb{Q}^{\mathrm{BS}}$. By the martingale central limit theorem, this convergence is equivalent to that the law of $\langle M\rangle_{T}$ under $\mathbb{Q}^{n}$ converges to a constant, which usually coincides with the limit of $V^{n}$. By specifying the rate of this convergence, we get the following asymptotic expansion result that is a simplified version of Theorem 2.5 and Corollary 2.6 of Fukasawa [5], where they are applied to many concrete examples including the fast mean reverting, regular perturbation and small vol-of-vol expansions.

Theorem 3.2 Suppose that $\liminf _{n \rightarrow \infty} V^{n}>0, \limsup _{n \rightarrow \infty} V^{n}<\infty$ and that there exists a positive sequence $\epsilon_{n}$ with $\lim _{n \rightarrow \infty} \epsilon_{n}=0$ such that the law of

$$
\left(\frac{M_{T}}{\sqrt{V^{n}}}, \frac{\langle M\rangle_{T}-V^{n}}{\epsilon_{n} V^{n} \sqrt{V^{n}}}\right)
$$

under $\mathbb{Q}^{n}$ converges to a 2-dimensional normal distribution with covariance $\alpha$. Suppose also that the $p$-th absolute moments of

$$
\frac{M_{T}}{\sqrt{V^{n}}}, \frac{\langle M\rangle_{T}-V^{n}}{\epsilon_{n} V^{n} \sqrt{V^{n}}},\left\{\int_{0}^{T} F_{s}^{2} \mathrm{~d} s\right\}^{-1}
$$

under $\mathbb{Q}^{n}$ are bounded for any $p>0$. Then, for any Borel function $f$ with $f \circ \exp$ 
being of polynomial growth,

$$
\mathbb{E}_{\mathbb{Q}^{n}}\left[f\left(S_{T}\right)\right]=\int_{\mathbb{R}} f\left(S_{0} \exp \left(\sqrt{V^{n}} z-\frac{V^{n}}{2}\right)\right) \phi_{n}(z) \mathrm{d} z+o\left(\epsilon_{n}\right),
$$

where

$$
\phi_{n}(z)=\phi(z)\left\{1+\frac{\alpha V^{n}}{2} \epsilon_{n}\left(\frac{z^{3}-3 z}{\sqrt{V^{n}}}-\left(z^{2}-1\right)\right)\right\}
$$

and $\phi$ is the standard normal density. Further for all $k \in \mathbb{R}$,

$$
\Sigma_{\mathrm{BS}}(k)=V^{n}\left(1+\alpha \epsilon_{n}\left(k+\frac{V^{n}}{2}\right)\right)+o\left(\epsilon_{n}\right) .
$$

Theorem 3.3 In addition to the assumptions in Theorem 3.2, if the law of

$$
S_{T} \epsilon_{n}^{-1}\left(\langle\log (S)\rangle_{T}-V^{n}\right)
$$

under $\mathbb{Q}^{n}$ is uniformly integrable, then

$$
\Sigma_{\mathrm{BS}}(k)=V^{n}\left(1+\frac{L^{n}}{2}\right)+L^{n} k+o\left(\epsilon_{n}\right),
$$

where $L^{n}$ is the MFIL;

$$
L^{n}:=\mathbb{E}_{\mathbb{Q}^{n}}\left[\left(\frac{S_{T}}{S_{0}}-1\right) \frac{\langle\log (S)\rangle_{T}}{V^{n}}\right] .
$$

Proof: The additional assumption is equivalent to that the law of

$$
\exp \left(M_{T}-\frac{1}{2}\langle M\rangle\right) \frac{\langle M\rangle_{T}-V^{n}}{\epsilon_{n} V^{n} \sqrt{V^{n}}}
$$

under $\mathbb{Q}^{n}$ is uniformly integrable. Therefore,

$$
L^{n}=\mathbb{E}_{\mathbb{Q}^{n}}\left[\frac{S_{T}}{S_{0}} \frac{\langle\log (S)\rangle_{T}-V^{n}}{V^{n}}\right]=\epsilon_{n} \sqrt{V^{n}} \mathbb{E}_{\mathbb{Q}^{n}}\left[\exp \left(\sqrt{V^{n}} N_{1}-\frac{V^{n}}{2}\right) N_{2}\right]+o\left(\epsilon_{n}\right),
$$

where $\left(N_{1}, N_{2}\right)$ follows the 2-dimensional normal distribution appeared in the assumptions of Theorem 3.2. Since the covariance between $N_{1}$ and $N_{2}$ is $\alpha$,

$$
L^{n}=\epsilon_{n} \alpha V^{n}+o\left(\epsilon_{n}\right) .
$$

The result then follows from Theorem 3.2. 


\section{The time-series of the MFIV and MFIL}

In this section we report some empirical evidence from the time-series of the MFIV and MFIL of the Nikkei stock average. We show that the MFIL reflected the fear of the market participants for the effect of a huge earthquake and tsunami which hit Japan on March 11, 2011. The joint dynamics of the MFIV and MFIL clearly distinguished the disaster and the financial crisis on 2008, the two different kinds of turmoil. Further we demonstrate a parametric estimation using YUIMA, an R package for estimations and simulations of stochastic differential equations. It indicates the existence of independent factors which drive the time-series of the MFIV and MFIL.

We use the representation obtained in the last section

$$
L_{0}=\frac{G_{0}}{V_{0}}-1=\frac{\int_{\mathbb{R}} \Sigma_{\mathrm{BS}}\left(g_{1}(z)\right) \Phi(\mathrm{d} z)}{\int_{\mathbb{R}} \Sigma_{\mathrm{BS}}\left(g_{2}(z)\right) \Phi(\mathrm{d} z)}-1 .
$$

See Fukasawa et al. [7] for the advantages of this type of formula compared to the CBOE's VIX type formula. Our data are from Nikkei NEEDS, the daily closing quote prices of call and put options traded on the Osaka Securities Exchange. We fix $T=30$ days $=30 / 365$ year and compute $G_{0}$ and $V_{0}$ for every day from 4 January 2006 to 21 October 2011. Since there is no option with maturity exactly $T=30$ days for every day, we use the option prices with the closest two maturities $T_{1}$ and $T_{2}$ to calculate the corresponding quantities $G_{0}\left(T_{i}\right)$ and $V_{0}\left(T_{i}\right)$ for $i=1,2$ and then interpolate them. For most of days $T_{1}<T<T_{2}$. After this interpolation, we set $L_{0}$ to be $G_{0} / V_{0}-1$. This idea of interpolation follows the CBOE's method for computing the VIX; however we introduce a slightly different interpolation formula in order to avoid unpleasant jumps of the time-series of $L_{0}$ which the CBOE's formula yields. We set

$$
\begin{aligned}
& \log \left(G_{0}\right)=\log \left(G_{0}\left(T_{1}\right)\right)+\left(\log \left(G_{0}\left(T_{2}\right)\right)-\log \left(G_{0}\left(T_{1}\right)\right)\right) \frac{\log (T)-\log \left(T_{1}\right)}{\log \left(T_{2}\right)-\log \left(T_{1}\right)} \\
& \log \left(V_{0}\right)=\log \left(V_{0}\left(T_{1}\right)\right)+\left(\log \left(V_{0}\left(T_{2}\right)\right)-\log \left(V_{0}\left(T_{1}\right)\right)\right) \frac{\log (T)-\log \left(T_{1}\right)}{\log \left(T_{2}\right)-\log \left(T_{1}\right)} .
\end{aligned}
$$




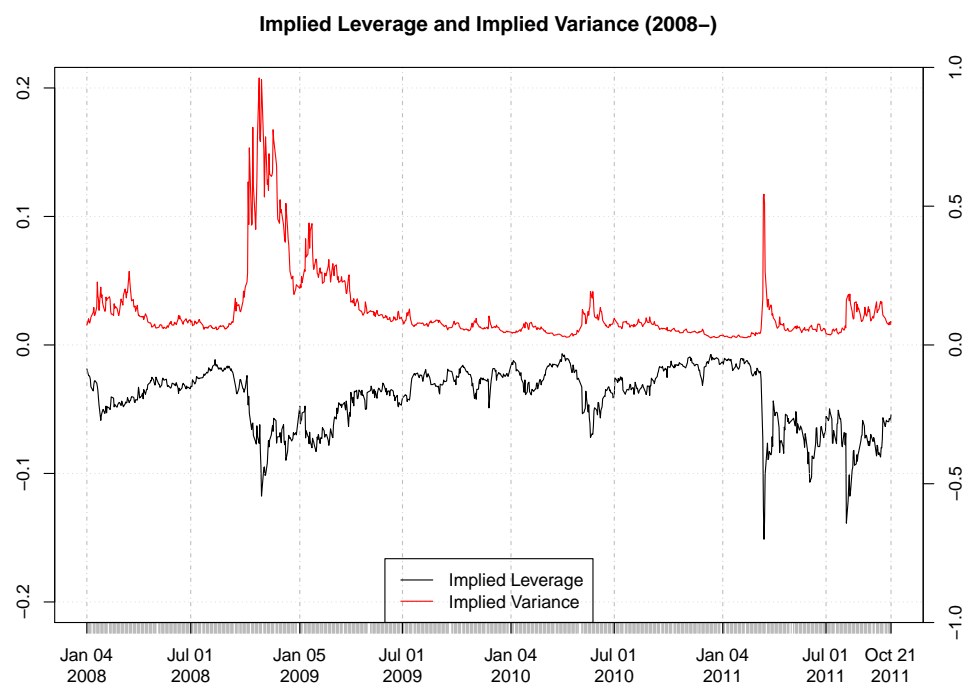

Figure 1: The time-series of the MFIL and MFIV

This is the log-linear interpolation, while the CBOE use the linear interpolation.

Figure 1 shows the time-series of the MFIL and MFIV divided by T. The MFIV divided by T, or Implied Variance, took its maximum 0.96 on 27 October 2008 in the middle of a financial crisis. The MFIL divided by $T$, or Implied Leverage, stayed negative in this period and took its minimum -0.15 on 16 March 2011, when the damage caused by an earthquake and tsunami which hit Japan on 11 March had been recognized in detail. During the financial crisis the MFIL was strongly negative as well but its reaction to the huge disaster was much stronger. In contrast the peak of the MFIV after 11 March did not reach its level for the financial crisis and it quickly came back to the usual level. Recall that the MFIV is, or more precisely its annualized square root is sometimes referred as the fear gauge due to its negative relation to the market return. If one was monitoring the MFIV only, he might conclude that the market's fear was over soon after the earthquake. On the other hand, as clearly seen from the 
Normal QQ plot: $\log (\mathrm{V})$

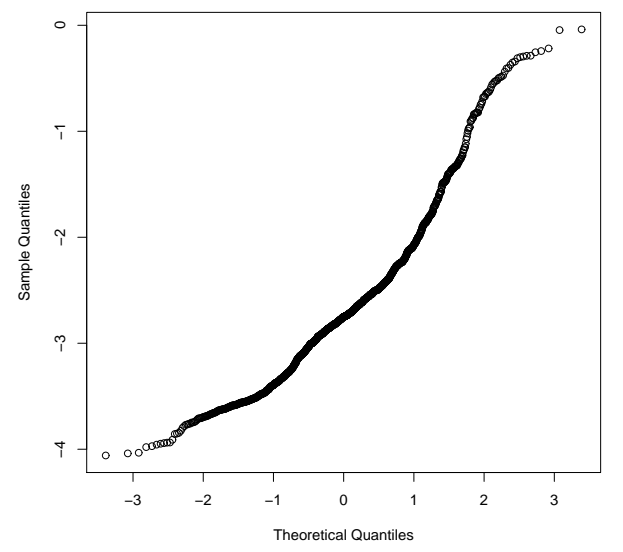

Normal QQ plot: Log(-L)

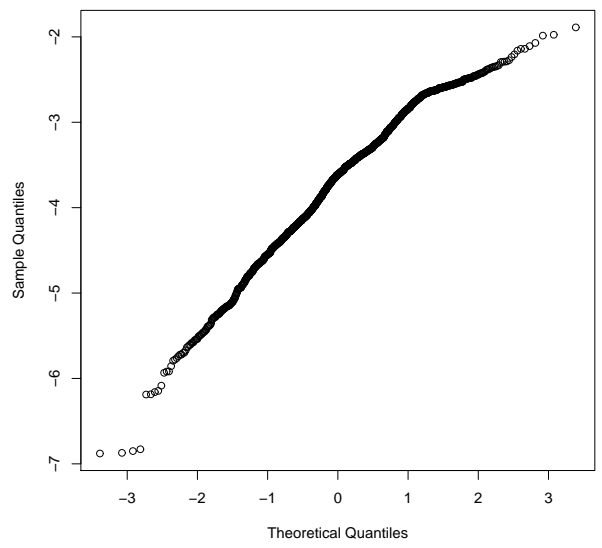

Figure 2: Normal QQ plots

figure, the MFIL have stayed at unusual level, which means that the negative effect of the disaster to the market sentiment was not over at all. The MFIL may be regarded as the fear gauge of the fear gauge in that it measures how strongly the fear gauge is correlated to the underlying market return. In light of the result in the previous section, the Black-Scholes implied volatility skew became more strongly negative after the earthquake. First both of the call and put option prices jumped up. Then the OTM call prices came back to the usual level soon, while the OTM put prices remained high. Consequently, the MFIL provides an important information which we cannot derive from the MFIV solely.

Figure 2 presents the normal QQ plots of the time-series $\log \left(V_{0} / T\right)$ and $\log \left(-L_{0} / T\right)$. These suggest that the ergodic distributions of the two processes are, if they exist, not so far from normal distributions. The most elementary diffusion process that has a normal distribution as its ergodic distribution is the OU process. Motivated by this observation, here we conduct a parametric 
estimation by assuming $X^{1}:=\log \left(V_{0} / T\right)$ and $X^{2}:=\log \left(-L_{0} / T\right)$ to follow

$$
\begin{aligned}
& \mathrm{d} X_{t}^{1}=\left(a_{1}+b_{1} X_{t}^{1}+c_{1} X_{t}^{2}\right) \mathrm{d} t+\sigma_{1} \mathrm{~d} W_{t}^{1}, \\
& \mathrm{~d} X_{t}^{2}=\left(a_{2}+b_{2} X_{t}^{1}+c_{2} X_{t}^{2}\right) \mathrm{d} t+\rho \mathrm{d} W_{t}^{1}+\sigma_{2} \mathrm{~d} W_{t}^{2}
\end{aligned}
$$

where $\left(W^{1}, W^{2}\right)$ is a standard Brownian motion. We use YUIMA, an R package for inference and simulations for stochastic differential equations, to obtain the following quasi-maximum likelihood estimates;

$$
\begin{aligned}
& \left(a_{1}, a_{2}, b_{1}, b_{2}, c_{1}, c_{2}, \sigma_{1}, \sigma_{2}, \rho\right) \\
& =(-10.26,-18.60,-6.10,4.90,1.59,-8.64,2.21,2.27,1.23)
\end{aligned}
$$

with standard deviation

$$
(4.33,5.07,1.91,2.24,1.67,1.95,0.04,0.04,0.06) \text {. }
$$

In particular the $99 \%$ confidence interval for $\sigma_{2}$ is $(2.17,2.37)$, which means that the value for $\sigma_{2}$ is significant, so that the MFIL contains a factor which is not explained by the movement of the MFIV. The standard deviations for the drift coefficients are large in contrast to those for the diffusion coefficients. This is consistent with what the the theory of high-frequency data analysis suggests. See for example Yoshida [12] for the detail of the quasi-likelihood estimation for diffusion processes. Due to the large standard deviation, the value for $c_{1}$ is not significant. We may therefore consider a reduced model

$$
\begin{aligned}
& \mathrm{d} X_{t}^{1}=\left(a_{1}+b_{1} X_{t}^{1}\right) \mathrm{d} t+\sigma_{1} \mathrm{~d} W_{t}^{1}, \\
& \mathrm{~d} X_{t}^{2}=\left(a_{2}+b_{2} X_{t}^{1}+c_{2} X_{t}^{2}\right) \mathrm{d} t+\rho \mathrm{d} W_{t}^{1}+\sigma_{2} \mathrm{~d} W_{t}^{2}
\end{aligned}
$$

and compare with the full model by CIC proposed by Uchida [11]. The CIC, standing for Contrast-based Information Criterion, is a criterion for model selection and defined as

$$
\text { CIC }=-2 l\left(\hat{\theta}_{\mathrm{qmle}}\right)+2 \operatorname{dim}(\Theta),
$$

where $l: \Theta \rightarrow \mathbb{R}$ is a quasi-log-likelihood function, $\hat{\theta}_{\text {qmle }}$ is the quasi-maximum likelihood estimator and $\Theta$ is the domain of unknown parameters. For the full model, $\operatorname{dim}(\Theta)=9$ and for the reduced model, $\operatorname{dim}(\Theta)=8$. The smaller the 
CIC, the better the model is expected to predict the distribution of the timeseries. Again by YUIMA, we get the quasi-maximum likelihood estimates

$$
\begin{aligned}
& \left(a_{1}, a_{2}, b_{1}, b_{2}, c_{2}, \sigma_{1}, \sigma_{2}, \rho\right) \\
& =(-12.61,-19.89,-4.75,5.66,-9.54,2.21,2.27,1.23)
\end{aligned}
$$

with standard deviation

$$
(3.57,4.88,1.29,2.10,1.72,0.04,0.04,0.06) \text {. }
$$

The CIC for the full model is -3080.59 and that for the reduced model is -3081.67. The CIC therefore suggests to select the latter. The $99 \%$ confidence interval for $\sigma_{2}$ is the same as before, so the value for $\sigma_{2}$ remains significant.

So far we confirmed that the MFIL conveys an additional information to the MFIV. More detailed empirical analysis is left for future research.

\section{Conclusion}

In this paper we have developed a rigorous formulation of robust replication theory for volatility derivatives. We have introduced several new derivatives, one of which is a swap contract on the quadratic covariation between an asset price and the model-free implied variance (MFIV) of the asset. It can be replicated in a model-free manner and its fair strike can be interpreted as a model-free measure for the covariance of the asset price and the realized variance. Based on this, we have proposed to call the fair strike the "model-free implied leverage" (MFIL). We have given a simple representation formula for the MFIL in terms of the Black- Scholes implied volatility. We have also shown its simple relation to the Black-Scholes implied volatility skew by an asymptotic method. Some explicit calculations under the Heston model have been given, and some empirical evidence from the time series of the MFIV and MFIL of the Nikkei stock average have been reported. Left for future research are extensions to semi-martingales with jumps, extensions to discrete-time rebalancing, 
managing discrete sets of strike prices for call and put options, applications to hedging other derivatives, modeling of the time-series of the MFIL, and empirical analysis for its ability to predict the realized leverage and market returns.

Acknowledgement: The author is grateful to anonymous Managing Editor and Referee for their suggestions to improve the quality of this paper. This work is supported by Japan Society for the Promotion of Science, KAKENHI (Grants-in-Aid for Scientific Research) Grant Numbers 22243021 and 24684006.

\section{References}

[1] Carr, P. and Lee, R. (2009): Volatility Derivatives, Annu. Rev. Financ. Econ. 1:1-21.

[2] Carr, P. and Lee, R. (2009): Put-Call Symmetry: Extensions and Applications, Math. Finance, 19, 523-560.

[3] Delbaen, F. and Schachermayer, W. (2006): The Mathematics of Arbitrage, Springer Finance.

[4] Fukasawa, M. (2011): Asymptotic analysis for stochastic volatility: Edgeworth expansion, Electronic J. Probab. 16, 764-791.

[5] Fukasawa, M. (2011): Asymptotic analysis for stochastic volatility: martingale expansion, Finance Stoch. 15, 635-654.

[6] Fukasawa, M. (2012): The normalizing transformation of the implied volatility smile, Mathematical Finance 22, 753-762.

[7] Fukasawa, M., Ishida, I., Maghrebi, N., Oya, K., Ubukata, M. and Yamazaki, K. (2011) Model-free implied volatility: from surface to index, International J. Theor. Appl. Finance 14, 433-463. 
[8] Gatheral, J. (2006): The Volatility Surface: A Practitioner's guide, Wiley Finance.

[9] Karandikar, R.L. (1995): On pathwise stochastic integration, Stochastic Process. Appl. 57 (1995), no. 1, 11-18.

[10] Neuberger, A. (2009): The Slope of the Smile, and the Comovement of Volatility and Returns, Available at SSRN: http://ssrn.com/abstract=1358863

[11] Uchida, M. (2010): Contrast-based information criterion for ergodic diffusion processes from discrete observations, Ann. Inst. Stat. Math. 62, 161-187.

[12] Yoshida, N. (2011): Polynomial type large deviation inequalities and quasilikelihood analysis for stochastic differential equations, Ann. Inst. Stat. Math. 63, 431-479. 